\title{
Site-directed spin-labeling of nucleotides and the use of in-cell EPR to determine long-range distances in a biologically relevant environment
}

\author{
Mykhailo Azarkh $^{1-3}$, Vijay Singh ${ }^{1-3}$, Oliver Okle ${ }^{1,2}$, Isabelle T Seemann ${ }^{1,2}$, Daniel R Dietrich ${ }^{1,2}$, Jörg S Hartig ${ }^{1,2}$ \& \\ Malte Drescher ${ }^{1,2}$ \\ ${ }^{1}$ Department of Chemistry, Konstanz Research School of Chemical Biology and Zukunftskolleg, University of Konstanz, Konstanz, Germany. ${ }^{2}$ Department of Biology, \\ Konstanz Research School Chemical Biology and Zukunftskolleg, University of Konstanz, Konstanz, Germany. ${ }^{3}$ Present addresses: Department of Molecular Physics, \\ Leiden University, Leiden, The Netherlands (M.A.), Department of Chemistry, Stanford University, Stanford, California, USA (V.S.). Correspondence should be addressed \\ to M.D. (malte.drescher@uni-konstanz.de).
}

Double electron-electron resonance (DEER) is an electron paramagnetic resonance (EPR) technique used to determine distance distributions in the nanometer range between spin labels by measuring their dipole-dipole interactions. Here we describe how in-cell DEER can be applied to spin-labeled DNA sequences to unravel their conformations in living cells by long-range distance measurements in cellula. As EPR detects unpaired electron spins only, diamagnetic molecules provide no background and do not reduce detection sensitivity of the specific signal. Compared with in-cell NMR spectroscopy, low concentrations of spin-labeled molecules can be used owing to the higher sensitivity of EPR per spin. This protocol describes the synthesis of the spin labels, their introduction in DNA strands, the injection of labeled DNA solutions in cells and the performance of in-cell EPR measurements. Completion of the entire protocol takes $\sim 20 \mathrm{~d}$.

\section{INTRODUCTION}

Elucidating the structure and dynamics of DNA, particularly of noncanonical DNA sequences such as those that form quadruplexes, is crucial for understanding their physiological function. Besides widely used techniques such as NMR or X-ray crystallography, sophisticated EPR spectroscopy methods have been successfully exploited for the structural probing of DNA sequences. Structural information obtained in in-cell studies is more useful than that obtained in in vitro experiments, as the cellular environment is characterized by factors and conditions (i.e., the presence of macromolecules, small molecules and crowding effects) that may have a crucial role in determining the biologically relevant conformation of the DNA sequence under investigation. Therefore, EPR spectroscopy in cellula is an important link between classical molecular biophysics and systems biology.

A prerequisite for the observation of macromolecules by EPR spectroscopy, however, is that they contain paramagnetic centers. Site-directed spin-labeling (SDSL) EPR enables the study of the structure and dynamics of DNA sequences, which do not contain native paramagnetic centers. Of particular interest are EPR techniques that enable researchers to measure broad intermolecular and intramolecular distance distributions; DEER, also referred to as pulsed electron double resonance, is most widely applied ${ }^{1}$.

Nitroxides are the usual spin labels in SDSL EPR ${ }^{1}$. These compounds are stable free radicals having the general formula $\mathrm{O}-\mathrm{NR}_{1} \mathrm{R}_{2}$, where the nitrogen atom is usually part of a heterocyclic ring. In nitroxides, the unpaired electron required for EPR detection is (de-)localized on the $\mathrm{N}-\mathrm{O}$ bond. The spin label can be attached covalently to larger molecules, for instance $\mathrm{DNA}^{2}$. Distance measurements between spin labels rely on dipole-dipole coupling between them, which is inversely proportional to the cube of the distance. DEER can be used to separate the dipole-dipole interaction from other contributions of the spin Hamiltonian, and it thereby provides information on distances in the $1.5-10-\mathrm{nm}_{\text {range }} 3$.
This protocol describes how to apply in-cell DEER to the determination of DNA conformations in living cells. In-cell EPR samples are prepared by injecting the spin-labeled macromolecule of interest into cells, for instance mature (stage VI) oocytes of Xenopus laevis.

\section{Comparison with other methods}

Förster resonance energy transfer (FRET) is widely used for studying distances in biomacromolecules. This technique requires the use of two mutually distinct, relatively bulky chromophores, the choice of which depends on the expected distance. By contrast, EPR distance measurements can be carried out using two much smaller, identical spin labels, the use of which makes it possible to maintain precision over a wide range of distances. Furthermore, depending on the labeling strategy adopted, both intermolecular and intramolecular distances can be measured. Distances between molecules can, in fact, be obtained from singly spin-labeled samples. Doubly spin-labeled molecules can instead be used to measure distances within molecules. When measuring intramolecular distances, it is good practice to conduct control experiments using singly spinlabeled macromolecules to identify and eliminate background intermolecular contributions to dipole-dipole coupling.

In comparison with in-cell $\mathrm{NMR}^{4,5}$, in-cell EPR has two major advantages: low concentrations of spin labels can be used because EPR sensitivity per spin is much higher than that of NMR, and diamagnetic molecules in the vicinity of the spin label will not reduce the detection sensitivity of the signal, as EPR detects only unpaired electron spins. The latter advantage is particularly notable as, in contrast to structural studies conducted on isolated macromolecules, in in-cell experiments the macromolecules under investigation are often found in close proximity to many different cellular components, which could otherwise be associated with high background signal and decreased detection sensitivity of the desired signals. 
Another advantage of in-cell EPR resides in the fact that, whereas solution-state NMR requires tumbling of the molecule under investigation, this attribute is not a prerequisite for in-cell EPR experiments. This is of particular importance when the macromolecule of interest might join large macromolecular complexes in cellula.

In-cell EPR distance measurements still have two major drawbacks, which they share with current state-of-the art in vitro DEER measurements: they are performed at low temperatures $(40-50 \mathrm{~K})$, after shock-freezing of the samples, and they rely on ensemble averaging. Although single-molecule sensitivity for routine experiments is currently out of reach in EPR spectrometry, so the pitfall of ensemble averaging cannot be avoided, ambient temperature distance measurements are a challenge; this is currently being addressed in order to move the field forward.

\section{Applications}

Possible applications of in-cell DEER can best be described by a brief survey of experiments already reported. The first in-cell DEER experiment has been reported by Igarashi et al. ${ }^{6}$ in 2010. Human ubiquitin was spin-labeled with pairs of spin labels at residues 20 and 35, and 35 and 52, respectively. Intramolecular spin-spin distances determined in cellula were similar to those measured in vitro, which suggests that no large structural differences existed between the ubiquitin derivatives used in an intracellular and an in vitro environment ${ }^{6}$.

In 2011, a model system consisting of a spin-labeled, 7-bp DNA double helix was delivered into Xenopus oocytes to demonstrate the feasibility and potential of conducting in-cell DEER experiments to study $\mathrm{DNA}^{7}$. The in-cell distance, $d$, between the spin-labeled terminal thymidines was determined to be $d=3.2 \mathrm{~nm}$, a value in agreement with that theoretically predicted, as well as with that experimentally measured in the isolated duplex in buffer. Moreover, broadening of the in-cell distance distribution indicated partial DNA melting7.

Krstic et al. ${ }^{8}$ investigated the structure of a 12-bp DNA double strand. This oligonucleotide was longer and had a higher relative content of G-C pairs, and thus higher stability, than the 7-bp duplex mentioned above, and in cellula it displayed the same conformation as it did in vitro. Aside from that attributed to intramolecular distances, a second broader component of the distance distribution was assigned to intermolecular distances and interpreted as evidence of end-to-end stacking of the DNA duplexes and of duplex ordering into semirigid rod-like structures ${ }^{8}$. Long-range distance constraints obtained by in-cell DEER for a 14-mer cUUCGg tetraloop hairpin RNA and the 27-mer neomycin-sensing riboswitch in Xenopus oocytes showed that this technique is also applicable to RNA sequences ${ }^{8}$.

Conducting in-cell DEER experiments on DNA sequences with a high degree of polymorphism is of particular interest. One such system is the human telomeric repeat, which, depending on subtle changes in environmental conditions, is known to adopt drastically different quadruplex conformations. Indeed, some evidence of the coexistence of different quadruplex conformations was brought to light by in vitro EPR experiments ${ }^{9}$. NMR spectra acquired in cellular extracts suggest that telomeric repeats possibly coexist in these extracts as an equilibrium mixture of different topologies; however, the low resolutions of in-extract NMR and in-cell NMR did not allow definite structural conclusions to be drawn ${ }^{10,11}$.
In-cell DEER was successfully used to study the folding of human telomeric quadruplexes upon microinjection of the unfolded sequence d[AGGG(TTAGGG) ${ }_{3}$ ] into living cells. The maxima in the distance distribution observed were assigned to the parallel propeller and the antiparallel basket conformations, whereas the presence of other possible quadruplex topologies, including intermolecular quadruplexes, was excluded ${ }^{12}$.

Therefore, not only can structural information on individual quadruplexes be inferred from EPR distance measurements but, more importantly, individual quadruplexes can be studied within the context of an extended sequence that better resembles the $3^{\prime}$ terminal, single-stranded overhang of vertebrate telomeric DNA. We studied G-quadruplex conformations within a sequence of three quadruplex units of human telomeric DNA by DEER ${ }^{13}$. In contrast with some individual G-quadruplexes, within the higherorder human telomeric sequence, we observed the formation of a $(3+1)$ hybrid structure ${ }^{13}$.

These applications of in-cell EPR distance measurements provide a foundation for future experiments that will enable researchers to follow biological processes in cells, in processes such as diffusion and interaction with proteins and other biologically relevant factors, and in chemical reactions. Studies that will apply in-cell EPR to probing structural aspects of nucleic acids through cell cycle progression are of particular interest.

\section{Experimental design}

Labeling strategy. The synthesis of doubly nitroxide-labeled oligonucleotides and their subsequent use in in vitro DEER analyses has already been described in a detailed protocol ${ }^{2}$. In addition, a body of literature on spin-labeling of oligonucleotides and EPR distance measurements is available $8,9,14-23$. The current protocol focuses on in-cell DEER of oligonucleotides. The main obstacle in conducting in-cell DEER experiments is the short half-life of the nitroxide spin labels in the cellular environment. The kinetic data on spin-label decay in cells can be described according to the Michaelis-Menten model, which suggests an enzymatic or enzyme-mediated nitroxide reduction process. The stability of nitroxide spin labels depends primarily on the size of the label's heterocyclic ring; five-membered rings have much higher stability in the intracellular environment than the six-membered rings, and thus the former are the preferred spin-label type for in-cell EPR experiments ${ }^{24}$. In the cellular environment of $X$. laevis oocytes, the 2,2,5,5-tetramethylpyrroline-1oxyl-3-acetylene (TPA) spin label has a half-life of over $20 \mathrm{~min}$ when attached to nucleic acids ${ }^{8,12}$.

In this protocol, we describe in detail the synthesis of the TPA nitroxide spin labels (Fig. 1) and the synthesis and purification of oligonucleotides, as there are some differences between the present approach and the protocol by Schiemann et al. ${ }^{2}$. We carry out DNA synthesis via conventional procedures, but we take care to minimize acidic conditions as much as possible, as these conditions promote unwanted reduction of the nitroxide radical to the corresponding amine ${ }^{25}$. In addition, the strategy we use to incorporate the spin labels differs in that we introduce the nitroxide spin label during the solid-phase DNA synthesis by direct coupling of TPAlabeled nucleoside phosphoramidites, whereas in the approach by Schiemann et al. ${ }^{2}, 5$-iodo-deoxyuridine is incorporated and a subsequent Sonogashira cross-coupling on the controlled pore glass (CPG) solid support is performed. 
Figure 1 | Synthesis of TPA-labeled 5'-DMTprotected 2'-deoxyuridine phosphoramidite (10). The synthesis of this compound is performed in accordance with procedures reported in the literature 37,38 , with some modifications. nBuLi, $n$-butyllithium; RT, room temperature.

The lengths of DNA strands synthesized via our protocol are similar to those typical of unmodified DNA. For example, we have synthesized doubly spin-labeled strands containing up to 12 repeats of the human telomeric hexamer sequence, for a total length of about $70 \mathrm{nt}$ (ref. 13). In principle, strands can be longer than $100 \mathrm{nt}$, but it should be noted that a small proportion of the spin labels are reduced at each round of coupling. Sequences should therefore be designed so that they are labeled at positions near the $5^{\prime}$ end of the strands rather than the $3^{\prime}$ end, as the extent of the reduction of the nitroxide will be diminished by this stratagem. Although DNA carrying reduced labels is removed during HPLC purification, excessive reduction will lower the overall yield of the DNA synthesis.

The injection volume per oocyte can vary between 30 and $100 \mathrm{nl}$ (cell volume is $\sim 1 \mu \mathrm{l}$ ), but the optimal value to avoid bursting of oocytes is $50 \mathrm{nl}$, which results in an 20 -fold dilution of the DNA solution in the cells. As the spherical oocytes will not occupy the entire space of the EPR tube (Fig. 2), the effective concentration will be even lower (by a factor of 1.3-1.5). These dilution effects require a several-millimolar starting DNA concentration.

The best labeling positions, which result in distance distributions that fall within the optimum distance range (1.5-6 nm), are identified by analyzing high-resolution DNA structures. For instance those obtained from the Protein Data Bank (PDB) database (http:// www.rcsb.org/pdb/) may be a good starting point. Nitroxide spin labels are small and have been shown to have minimal effects on DNA structures ${ }^{26,27}$. However, as for all labeling techniques, control experiments aimed at excluding distortions of the original DNA conformation due to the attachment of the label are essential for SDSL.

In-cell DEER is often performed to elucidate the conformation of the DNA. In this case, only distances within this DNA sequence are of interest. The contribution of distances to neighboring molecules should therefore be suppressed. If singly labeled DNA sequences are available, we strongly recommend that the intermolecular contribution to dipole-dipole interactions be measured separately to derive an experimental background function. In principle, background data should be individually measured for both labeled positions of a doubly labeled sample. Only in cases in which no experimental background function from singly labeled molecules is available should a theoretical background function be used.

Microinjection. Although microinjections can be performed on many cells, because cell-injection procedures are time-consuming and elaborate, the number of cells manipulated should be as small as possible. Xenopus oocytes are ideal cells for this purpose, as they are large ( $1 \mu \mathrm{l}$ in volume) and thus only 60 are required for an incell EPR sample. Moreover, Xenopus oocytes are well characterized and widely available, with injection protocols well established and easily performed. DNA can be injected into the animal hemisphere cytoplasm of mature (stage VI) Xenopus oocytes, in which nucleus-like conditions exist. These oocytes resemble, in fact, the typical eukaryotic cell: for instance, the assembly around the DNA of structures contains lamin nuclear proteins and a double bilayer nuclear membrane is present, which contains nuclear pore complexes ${ }^{28,29}$.

Oocytes are usually injected using pulled glass needles mounted on a pneumatic or oil-driven injection device; each oocyte must be injected under a dissecting microscope to enable visual alignment with the tip of the needle. As an alternative, a robotic device can be used $^{30}$. Proper animal care and safety protocols must be followed at all times. Check with your local animal care facility or appropriate institutional official to obtain the necessary training and licenses. Alternatively, surgically extracted ovaries from mature frogs are commercially available.

As mentioned above, the nitroxide spin-label TPA has a relatively long in-cell half-life of, for instance, $29 \pm 7 \mathrm{~min}$ when attached to the human telomeric repeat ${ }^{12}$. Consequently, the experimenter must choose an optimum incubation time that allows intracellular DNA

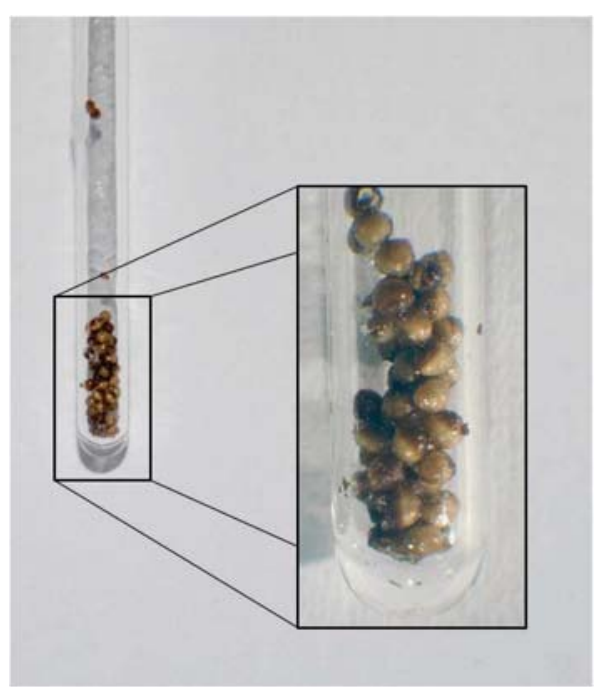

Figure $2 \mid X$. laevis oocytes in the EPR tube (4-mm outer diameter). 
Figure 3 | DEER experiment. (a) DEER pulse sequence. The observer pulse sequence is shown in the upper section of the figure. The positions of the primary and of the refocused echo are indicated by bell-like dashed curves. $\tau_{1}$ corresponds to the delay between the $\pi / 2$ pulse and the first $\pi$ pulse, and $\tau_{2}$ is the delay between the primary spin echo and the second $\pi$ pulse. The lower section of the figure shows the pump sequence, where $t$ defines the temporal position of the pump pulse. The origin of this time scale is given by the time of the primary echo (dotted line). (b) Nitroxide EPR spectrum in $X$ band; the pump pulse is applied at the maximum, whereas the observer frequency is shifted by $67 \mathrm{MHz}$. $B_{\mathrm{a}}$ is the applied magnetic field. (c) Resonator profile, the pump frequency corresponds to the resonator frequency. $V_{\mathrm{mw}}$ is the microwave frequency.

conformations to reach equilibrium while avoiding the reductive quenching of most of the nitroxide spin labels. After incubation, samples must be shock-frozen to trap the adopted conformations for in-cell distance measurements and to prevent further reduction of nitroxides.

Reasonable DEER time traces can be acquired in samples in which as many as $50 \%$ of spin labels are quenched ${ }^{8}$. Shock-freezing of the samples after different incubation times, followed by the collection of continuous wave (CW)-EPR spectra, enables the quantification of the rate of nitroxide reduction ${ }^{6,8,12}$. The lower limit of the proper incubation time, the minimum time needed for the macromolecule to reach a conformational equilibrium, can be roughly estimated by in vitro measurements (UV-visible and circular dichroism spectroscopy or FRET) ${ }^{12}$.

In-cell EPR. Frozen, noninjected Xenopus oocytes show an intrinsic CW-EPR signal, which overlaps with the central $m_{\mathrm{I}}=0$ line of the nitroxide spectrum ${ }^{7}$. For samples with a final intracellular concentration of $\sim 200 \mu \mathrm{M}$ of injected spin-labeled DNA, this intrinsic signal is $\sim 50$ times weaker than the signal of the nitroxide. As a background correction is performed for DEER measurements, the intrinsic cellular EPR signal is eliminated during this data processing step by dividing the in-cell DEER curve of doubly labeled DNA by the in-cell DEER curve of the corresponding singly labeled DNA.

The dead time-free four-pulse DEER sequence is shown in Figure 3. The experimenter has to choose the length of the measured dipolar evolution, which is approximately given by $t_{\max } \approx \tau_{2}$ (where $t_{\max }$ is the maximum dipolar evolution time and $\tau_{2}$ is the separation time between the primary echo and the second $\pi$-pulse of the observer sequence). The required $t_{\max }$ depends on the expected value of the distance to be measured. With $t_{\max }=2 \mu \mathrm{s}$, the shape of the distance distribution is reliable up to a distance of $3 \mathrm{~nm}$ (reliable distribution limit). The mean distance and the width

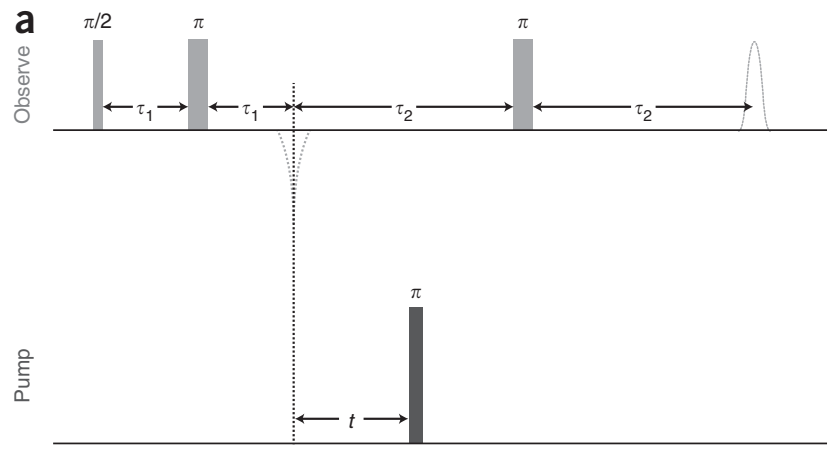

b

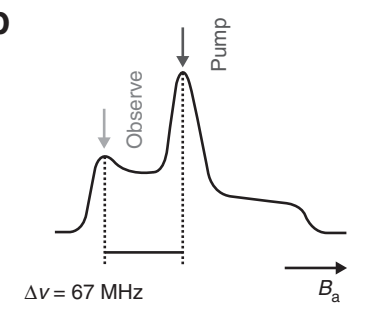

C

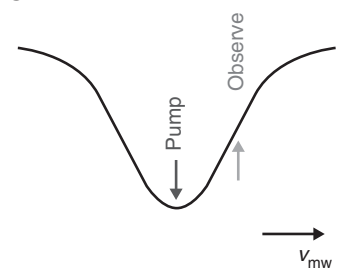

of its distribution are reliable up to a distance of $4 \mathrm{~nm}$ (reliable width limit), whereas the mean distance, but not the distribution width, is reliable between 4 and $5 \mathrm{~nm}$ (reliable mean distance limit). Beyond $5 \mathrm{~nm}$, no reliable mean value can be determined. All these limits scale with the cubic root of $t_{\max }$ (ref. 31 ).

Distance distributions can be computed from the DEER curve. A sketch of the procedure is given in Figure 4. The underlying mathematical problem is (moderately) ill posed, in that small variations in the experimental data (e.g., noise) can cause large variations in the distance distribution. Data should be acquired with as good a signal-to-noise ratio as possible. Reproducing results for a given sample is good research practice.

A good compromise between high resolution and limited influence of experimental noise must be reached during data analysis. This objective is usually achieved by Tikhonov regularization ${ }^{32}$, which makes data analysis of in-cell DEER experiments rather complex. However, the most widely used program package in the field using Tikhonov regularization is available free of charge at http://www.epr.ethz.ch/software, and it enables even nonspecialists to extract distance distribution curves ${ }^{33}$. This program package includes pre-processing tools to correct for phase errors and displacements of the time origin of the DEER curve and to separate the intramolecular interactions of interest from the intermolecular background contributions; the package also includes a very scholarly and detailed manual. a

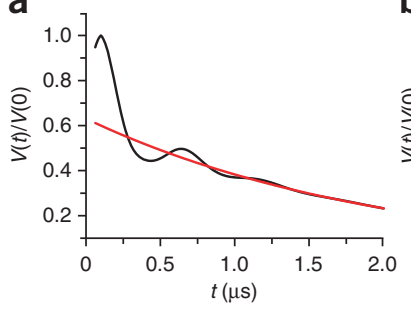

b

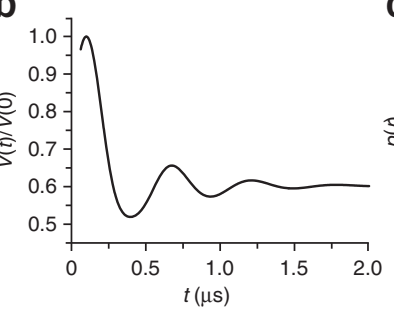

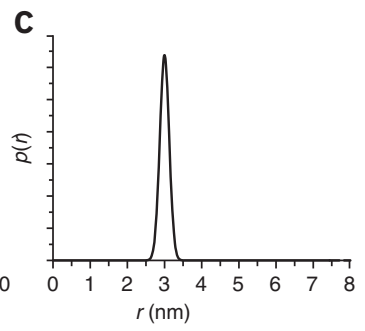

Figure 4 | Schematic representation of data analysis. (a) Black line, DEER raw data in terms of normalized echo intensity $V(t)$ versus pump pulse position $t$. Red line, background due to intermolecular interactions. (b) Form factor following background correction. (c) Distance distribution as obtained by Tikhonov regularization. $p(r)$ is the probability to find a distance within an interval dr centered around $r$. 


\section{MATERIALS \\ REAGENTS}

I CAUTION Most of the reagents for the DNA synthesizer are harmful, corrosive and highly flammable. Pay attention to the symbols on the bottles and wear protective clothing, eye protection and face protection when changing reagents on the synthesizer.

-2,2,6,6-Tetramethyl-4-piperidone (Sigma-Aldrich, cat. no. 459119)

- N,O-Dimethylhydroxylamine hydrochloride (Sigma-Aldrich,

cat. no. 459119)

- meta-Chloroperbenzoic acid (mCPBA; Sigma-Aldrich, cat. no. 273031)

! CAUTION mCPBA is flammable and toxic; wear protective gloves and a mask.

- Diisopropylamine (Sigma-Aldrich, cat. no. 386464)

-5-Iodo-2'-deoxyuridine (Sigma-Aldrich, cat. no. I7125)

- Copper(I) iodide (CuI; Sigma-Aldrich, cat. no. 215554) $\Delta$ CRITICAL CuI is light sensitive; store the compound in a dark (or cover with black paper) and dry place.

- Tetrakis(triphenylphosphine)palladium $(0)\left(\mathrm{Pd}\left(\mathrm{PPh}_{3}\right)_{4}\right.$; Sigma-Aldrich, cat. no. 216666)

- (Chloromethyl)triphenylphosphonium chloride $\left(\mathrm{ClCH}_{2} \mathrm{P}(\mathrm{Cl})\left(\mathrm{C}_{6} \mathrm{H}_{5}\right)_{3}\right.$; Sigma-Aldrich, cat. no. 377015)

- $n$-Butyllithium in hexane, 1.6 M (Sigma-Aldrich, cat. no. 186171))

I CAUTION $n$-Butyllithium is sensitive to moisture and air. It reacts violently with water, liberating extremely flammable gases. Wearing protective gloves during the transfer of solutions containing $n$-butyllithium is highly recommended.

- 4,4' -(Dimethoxy)trityl chloride (DMT-Cl; Sigma-Aldrich, cat. no. 100013)

-4-(Dimethylamino)pyridine (DMAP; Sigma-Aldrich, cat. no. 522805)

- Diisobutylaluminum hydride (DIBAL; Sigma-Aldrich, cat. no. 190306)

I CAUTION DIBAL is highly sensitive to moisture and air; it releases

flammable gases upon contact with water.

- N,N-diisopropylethylamine (DIPEA; Sigma-Aldrich, cat. no. 387649)

- 2-Cyanoethyl N,N-diisopropylchlorophosphoramidite (Sigma-Aldrich, cat. no. 302309) $\Delta$ CRITICAL This reagent is highly sensitive to air and moisture; open and handle the bottle in the presence of argon gas.

- Dichloromethane, puriss; absolute; over molecular sieve (DCM; Sigma-Aldrich, cat. no. 270997)

- Tetrahydrofuran, puriss; absolute; over molecular sieve (THF,

Sigma-Aldrich, cat. no. 401757)

- N,N-Dimethylformamide, puriss; absolute; over molecular sieve

(DMF, Sigma-Aldrich, cat. no. 227056)

- Pyridine, absolute; over molecular sieve (Sigma-Aldrich, cat. no. 270970; note that DCM, THF, pyridine and DMF are obtained in Sure/Seal bottles with a septum for syringe transfer and are used as received without further purification)

- Diethyl ether, anhydrous (Et ${ }_{2}$ O; Sigma-Aldrich, cat. no. 296082)

- Triethylamine, purum ( $\mathrm{Et}_{3} \mathrm{~N}$; Riedel deHaen, cat. no. 16304)

- Calcium chloride, anhydrous, granular $\left(\mathrm{CaCl}_{2}\right.$; Sigma-Aldrich,

cat. no. C1016)

- Sodim sulfate, anhydrous, granular $\left(\mathrm{Na}_{2} \mathrm{SO}_{4}\right.$; Sigma-Aldrich,

cat. no. 239313)

- Bromine $\left(\mathrm{Br}_{2}\right)$ ! CAUTION Bromine is toxic and causes burns; therefore, wear protective gloves, protective clothing, eye protection and face protection.

- Methanol

- Acetic acid (AcOH; Merck)

- Sodium hydroxide $(\mathrm{NaOH})$

- Hydrochloric acid $(\mathrm{HCl})$

- Ethyl acetate (EtOAc)

- Hexane

- Silica gel

- DNA phosphoramidites (Proligo Reagents, cat. nos. A111032 for dA,

T111032 for dT, C113032 for dC and G115032 for dG)

- Acetonitrile for DNA synthesis (J.T. Baker, cat. no. 8144)

- Packing material for columns (CPG) with the first base attached via an LCAA linker, 1,000 $\AA, 1 \mu \mathrm{mol}$ scale (3Prime, cat. nos. CPG-100-N-DA-Y-1 for dA, CPG-100-N-DG-X-1 for dG, CPG-100-N-DT-Y-1 for dT and CPG-100-

N-ACC-X-1 for dC)

- Ammonium hydroxide solution, 28-30\% (wt/wt) $\mathrm{NH}_{3}$ basis (Sigma-Aldrich, cat. no. 221228)! CAUTION Ammonium hydroxide causes burns.

- HPLC buffer: 0.1 M triethylammonium acetate (TEAA), pH 7.5
- Acetonitrile (HPLC grade)

-X. laevis frog, 3-4-year-old female ! CAUTION All experiments must be performed in accordance with relevant animal handling and care guidelines and regulations.

- Ethyl 3-aminobenzoate, $0.1 \%$ (Acros Organics)

- Collagenase type IA, 0.1\% (Sigma, cat. no. C-9891)

- Modified Barth's saline (MBS) medium, 10×: $880 \mathrm{mM} \mathrm{NaCl}, 10 \mathrm{mM} \mathrm{KCl}$,

$10 \mathrm{mM} \mathrm{MgSO}_{4}, 50 \mathrm{mM}$ HEPES (pH 7.8), $25 \mathrm{mM} \mathrm{NaHCO}_{3}$

- Ice

- Liquid nitrogen! CAUTION Wear protective goggles and gloves while

working with liquid nitrogen.

- Dry ice

- Distilled water (Millipore)

Solutions for the DNA syntheziser

- Deblock-TCA (trichloroacetic acid in $\mathrm{CH}_{2} \mathrm{Cl}_{2}$; J.T. Baker, cat. no. 9448)

- Capping A ( $N$-methyl imidazole; J.T. Baker, cat. no. 9497)

- Capping B (acetic anhydride; J.T. Baker, cat. no. 9485)

- Oxidizer (iodine; J.T. Baker, cat. no. 9488)

- Activator (5-(ethylthio)-1H-tetrazole, 0.25 M in acetonitrile; Azco Biotech, cat. no. 56-0001-34)

- Acetone

\section{EQUIPMENT}

- Teflon-coated magnetic stir bars

- Plastic syringes (all polypropylene)

- Disposable syringe needles

- Dripping funnel

- Filter paper

- Buchner funnel

- Rotary evaporator

- Vacuum pump

- Hot plate

- Rubber septum

- Nitrogen gas

- Argon gas

- ABI 394 DNA/RNA synthesizer or another commercial DNA synthesizer

- HLPC purification: we use a Prominence HPLC (Shimadzu)

instrument equipped with a Nucleosil-100-5 C18 column $(250 \times 4 \mathrm{~mm}$, Macherey-Nagel)

- DNA purification: filters to remove CPG beads (Sartorius Stedim Biotech, Ministart RC 15, cat. no. 17762)

- Removing solvents: SpeedVac (Eppendorf concentrator plus)

- DNA quantification: Bruker Esquire 3000plus electrospray ionization-mass spectrometry (ESI/MS) spectrometer

- Surgical instruments (sterile: scalpel, forceps, scissors, surgical thread)

- Pasteur pipettes

- Drummond Digital Microdispenser 500 system (Drummond Scientific)

- Bruker ELEXSYS E580 pulsed EPR spectrometer operating in X-band equipped with a dielectric resonator (MD4-W1); a unit for electron double resonance (ELDOR) providing a second microwave frequency; and helium gas flow system (CF 935) and temperature control system (ITC 502), both from Oxford Instruments

- High-quality quartz glass EPR tubes (4-mm outer diameter, Bruker)

- Lyophilizer (Lyovac GT2, Leybold-Heraeus)

- Weighing paper

- Aluminum foil

- Round-bottom flasks

Software

- MATLAB (MathWorks)

- DeerAnalysis ${ }^{33}$

\section{REAGENT SETUP}

Anhydrous $\mathrm{Et}_{3} \mathrm{~N}$ Add calcium chloride (50-60 g per 1 liter) to $\mathrm{Et}_{3} \mathrm{~N}$ (purum) and store it at room temperature $\left(\sim 22^{\circ} \mathrm{C}\right)$. It can be stored safely for 6 months. Aqueous $\mathrm{NaOH}, \mathbf{3 0} \%$ Dissolve $30 \mathrm{~g}$ of $\mathrm{NaOH}$ in 1 liter of Millipore water and store it at room temperature. It can be stored safely for 6 months. HCl, 2 M Slowly add $34.33 \mathrm{ml}$ of concentrated $36 \%$ (vol/vol) $\mathrm{HCl}(11.65 \mathrm{M}$ ) to $200 \mathrm{ml}$ of Millipore water under constant stirring; store it at room temperature. It can be stored safely for 6 months.

HPLC buffer, 0.1 M TEAA For 1 liter of buffer, add $5.7 \mathrm{ml}$ of AcOH (100\%, p.a.; Merck) and $13.9 \mathrm{ml}$ of $\mathrm{Et}_{3} \mathrm{~N}$ to $980 \mathrm{ml}$ of Millipore water. Adjust the $\mathrm{pH}$ to 7.5 with $\mathrm{AcOH}$ or $\mathrm{Et}_{3} \mathrm{~N}$. It can be stored safely at $4{ }^{\circ} \mathrm{C}$ for 6 months. 
MBS medium, $1 \times$ MBS $(1 \times)$ medium is prepared from $10 \times$ MBS medium immediately before use, and it is supplemented with $\mathrm{CaCl}_{2}$ to a final concentration of $700 \mu \mathrm{M} \mathrm{CaCl}_{2}$. MBS $(1 \times)$ medium can be used for the storage and manipulation (injection) of the Xenopus oocytes.

Defolliculation medium Defolliculation medium is $1 \times$ MBS medium supplemented with collagenase type IA to a final concentration of $0.1 \%(\mathrm{wt} / \mathrm{vol})$ collagenase type IA.

\section{EQUIPMENT SETUP}

DNA synthesizer Prepare the synthesizer by installing all the required phosphoramidites and reagents. Check that all installed reagents are fresh and sufficient for the synthesis. $\triangle$ CRITICAL Note that the guanosine phosphoramidite easily crystallizes in the tube; therefore, flushing tubes after synthesis is advisable.
EPR spectrometer Cooling with liquid helium is useful because it optimizes sensitivity, but it is not a prerequisite for these measurements. Alternatively, liquid nitrogen can be used.

EPR resonator The use of a dielectric resonator (MD4-W1, Bruker) offers a larger sample volume compared with the split ring resonator (MS3, Bruker) often used for conventional DEER experiments. A larger sample volume is preferable because the small injected volume limits the achievable overall concentration, whereas the available total sample volume is generally not restricted. I CAUTION Switch the spectrometer to standby when you remove or change EPR resonators.

Software Install DeerAnalysis in your MATLAB environment by following the instructions in the user manual.

\section{PROCEDURE}

Synthesis of 3,5-dibromo-2,2,6,6-tetramethylpiperidin-4-one hydrobromide (2) $\bigcirc$ TIMING 2 d

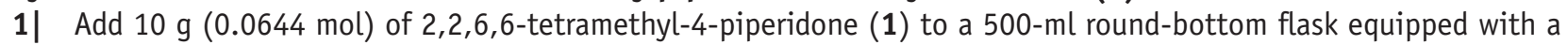
magnetic stir bar. Slowly add glacial $\mathrm{AcOH}(39 \mathrm{ml})$ under water bath cooling conditions.

2) Add a solution of $\mathrm{Br}_{2}(6.6 \mathrm{ml}, 0.128 \mathrm{~mol})$ in $\mathrm{AcOH}(28 \mathrm{ml})$ dropwise to the $500-\mathrm{ml}$ round-bottom flask via a dripping funnel over a period of $6 \mathrm{~h}$.

3| Continue stirring the reaction mixture at room temperature for $24 \mathrm{~h}$.

4| Filter the reaction mixture to obtain a solid product. Wash the solid with $20 \mathrm{ml}$ of AcOH, then with $20 \mathrm{ml}$ of $\mathrm{H}_{2} \mathrm{O}$ and finally three times with $20 \mathrm{ml}$ of $\mathrm{Et}_{2} \mathrm{O}$. Completely dry the product 2 in vacuo to obtain an off-white powder. Yield: $23.1 \mathrm{~g}(91 \%)$.

PAUSE POINT Product 2 can be stored safely at $4{ }^{\circ} \mathrm{C}$ for $4-5$ months.

\section{Synthesis of 3-( $N$-methoxy- $N$-methylcarbamoyl)-2,2,5,5-tetramethylpyrroline (3) TIMING $2 \mathrm{~d}$}

5| Cool a solution of $\mathrm{N}, 0$-dimethylhydroxylamine hydrochloride $(2.97 \mathrm{~g}, 30 \mathrm{mmol})$ in $\mathrm{H}_{2} \mathrm{O}(20 \mathrm{ml})$ by placing the roundbottom flask containing it in an ice bath and gradually add $\mathrm{Et}_{3} \mathrm{~N}(14.8 \mathrm{ml}, 107 \mathrm{mmol})$. Continue stirring for a few minutes at the same temperature (ice bath).

6| Remove the ice bath and stir the reaction mixture vigorously. Add compound 2 (10 g, $25 \mathrm{mmol})$ from Step 4 in small portions over $6 \mathrm{~h}$ and continue stirring overnight at room temperature.

7| Heat the reaction mixture to $50^{\circ} \mathrm{C}$ and continue stirring for $6 \mathrm{~h}$.

8| Cool the reaction mixture to room temperature and adjust its $\mathrm{pH}$ to 9.3 with $30 \%$ (wt/vol) $\mathrm{NaOH}$ (aqueous).

9| Extract the reaction mixture with EtOAc $(4 \times 20 \mathrm{ml}$; i.e., four washes of $20 \mathrm{ml}$ each). Separate the phases and dry the combined organic phase over anhydrous $\mathrm{Na}_{2} \mathrm{SO}_{4}$. Stir for a few minutes and filter the solid $\mathrm{Na}_{2} \mathrm{SO}_{4}$. Evaporate the solvent with a rotary evaporator at $30^{\circ} \mathrm{C}$.

10| Purify the crude reaction mixture containing product 3 by salt-desalt strategy: dissolve the crude product in EtOAc $(20 \mathrm{ml})$ and add an equal volume of $2 \mathrm{M} \mathrm{HCl}$ in order to obtain the hydrochloride salt of the pyrrole amine.

11 Separate the aqueous layer (impurities remain in the organic phase and the product remains in the aqueous layer). Adjust the $\mathrm{pH}$ of the aqueous layer to 9.3 with $30 \%$ (wt/vol) $\mathrm{NaOH}$ (aqueous) while keeping the separation mixture in an ice bath (this step produces the free amine from the hydrochloride salt).

12| Extract product 3 with EtOAc $(3 \times 20 \mathrm{ml})$. Separate the phases and dry the combined organic phase over anhydrous $\mathrm{Na}_{2} \mathrm{SO}_{4}$. Stir the mixture for a few minutes and filter off the solid $\mathrm{Na}_{2} \mathrm{SO}_{4}$. Evaporate the solvent with a rotary evaporator at $30{ }^{\circ} \mathrm{C}$ to isolate the product. Yield: $3.45 \mathrm{~g}(65 \%)$.

PAUSE POINT Product 3 can be stored safely at $4{ }^{\circ} \mathrm{C}$ for $3-4$ weeks. 
Synthesis of 3-(N-methoxy- $N$-methylcarbamoyl)-2,2,5,5-tetramethyl-1-oxy-pyrroline (4) $\bigcirc$ TIMING $1 \mathrm{~d}$

13| Dissolve $2.80 \mathrm{~g}(13.2 \mathrm{mmol})$ of compound 3, from Step 12, in DCM (20 ml) in a 100-ml round-bottom flask equipped with a magnetic stir bar; cool the flask by placing it in an ice bath.

14| Slowly add $4.52 \mathrm{~g}(26.4 \mathrm{mmol})$ of mCPBA and continue stirring for $3 \mathrm{~h}$.

15 Quench the reaction by adding $10 \mathrm{ml}$ of ice-cold water to it.

16| Extract the product with $\mathrm{DCM}(3 \times 15 \mathrm{ml})$. Collect and combine the organic phases and dry them over anhydrous $\mathrm{Na}_{2} \mathrm{SO}_{4}$. Stir the mixture for a few minutes and filter the solid $\mathrm{Na}_{2} \mathrm{SO}_{4}$. Evaporate the solvent with a rotary evaporator at $30{ }^{\circ} \mathrm{C}$ to isolate the crude product.

17| Purify the crude product over a silica gel column $(\sim 3 \mathrm{~cm}$ internal diameter $\times 20 \mathrm{~cm}$ length) and elute product 4 with EtOAc:hexane (80:20, vol/vol). Yield: $1.86 \mathrm{~g}(62 \%)$.

PAUSE POINT Product 4 can be stored safely at $4{ }^{\circ} \mathrm{C}$ for $3-4$ weeks.

Synthesis of 3-formyl-2,2,5,5-tetramethyl-1-oxy-pyrroline (5) $\bigcirc$ TIMING 1 d

18| Dissolve $1.84 \mathrm{~g}(8.09 \mathrm{mmol})$ of compound 4 (from Step 17) in anhydrous $\mathrm{Et}_{2} \mathrm{O}(10 \mathrm{ml})$ in a 50 - $\mathrm{ml}$ round-bottom flask equipped with a magnetic stir bar and continue stirring. Cool the mixture to $-78{ }^{\circ} \mathrm{C}$ by placing the round-bottom flask in an acetone-dry ice bath.

19| Add, dropwise, $10 \mathrm{ml}$ of a $1.0 \mathrm{M}(10 \mathrm{mmol})$ solution of DIBAL in hexane to the reaction mixture over 30 min under nitrogen atmosphere.

20| Continue stirring for $15 \mathrm{~min}$. Slowly pour the reaction mixture into a 10-ml, $2 \mathrm{M} \mathrm{HCl}$ solution that had been precooled to $0^{\circ} \mathrm{C}$ by placing the round-bottom flask that contains it in an ice-water bath.

$\Delta$ CRITICAL STEP Use an ice bath to cool the reaction mixture; strictly monitor the temperature of this step because a rise in temperature will result in poor quality and low yield of the product.

21 Slowly warm the reaction mixture to room temperature and extract the aqueous phase with EtOAc $(4 \times 15 \mathrm{ml})$.

22 Collect and combine the organic phases and dry them over anhydrous $\mathrm{Na}_{2} \mathrm{SO}_{4}$, stirring the mixture for a few minutes. Filter the solid $\mathrm{Na}_{2} \mathrm{SO}_{4}$ and evaporate the solvent using a rotary evaporator at $30^{\circ} \mathrm{C}$ to isolate the crude product.

Purify the crude product over a silica gel column $(\sim 3 \mathrm{~cm}$ internal diameter $\times 25 \mathrm{~cm}$ length) by elution with Et0Ac:hexane (10:90, vol/vol) to obtain product 5 as a light yellow solid. Yield: $1.17 \mathrm{~g}(86 \%)$.

PAUSE POINT Product 5 can be stored safely at $4^{\circ} \mathrm{C}$ for $3-4$ weeks.

Synthesis of 3-(2-chloroethyl)-2,2,5,5-tetramethyl-1-oxy-pyrroline (6) $\bigcirc$ TIMING $1 \mathrm{~d}$

23. Add $\mathrm{ClCH}_{2} \mathrm{P}(\mathrm{Cl})\left(\mathrm{C}_{6} \mathrm{H}_{5}\right)_{3}(8.2 \mathrm{~g}, 24 \mathrm{mmol})$ to $40 \mathrm{ml}$ of THF in a $100-\mathrm{ml}$ round-bottom flask equipped with a magnetic stir bar. Cool the reaction mixture to $-78{ }^{\circ} \mathrm{C}$ by placing the reaction flask in an acetone-dry ice bath.

24 Seal the flask with a rubber septum and add to it, dropwise, $13.23 \mathrm{ml}(21.8 \mathrm{mmol})$ of a $1.6 \mathrm{M}$ solution of $n$-butyllithium in hexane under nitrogen atmosphere.

$\triangle$ CRITICAL STEP Protect the reaction mixture from moisture and handle it under inert atmosphere, or the activity of $n$-butyllithium will be quenched.

25| Gradually raise the temperature to $-40^{\circ} \mathrm{C}$ by decreasing the addition of dry ice in an acetone bath and stir the reaction mixture at $-40{ }^{\circ} \mathrm{C}$ for $30 \mathrm{~min}$.

26| Recool the reaction mixture to $-78^{\circ} \mathrm{C}$ via the acetone-dry ice bath and add to it a $10-\mathrm{mL}$ THF solution of product 5 dropwise with a syringe $(1.0 \mathrm{~g}, 5.95 \mathrm{mmol}$ from Step 22).

27| Remove the acetone-dry ice bath and replace it with an ice-water bath. Continue stirring for 30 min.

28| Quench the reaction mixture by adding to it $5 \mathrm{ml}$ of ice-cold water. 
29| Remove the reaction volatiles in vacuo. To the residue obtained, add $15 \mathrm{ml}$ of water, and then extract the resulting mixture with EtOAc $(3 \times 20 \mathrm{ml})$. Combine the organic layers and dry them over anhydrous $\mathrm{Na}_{2} \mathrm{SO}_{4}$. After a few minutes of stirring, filter the solid $\mathrm{Na}_{2} \mathrm{SO}_{4}$ and concentrate it in vacuo. Purify the crude product over a silica gel column $(\sim 3 \mathrm{~cm}$ internal diameter $\times 25 \mathrm{~cm}$ length), eluting it with EtOAc:hexane (10:90, vol/vol) to isolate compound 6 as a yellow oil. Yield: $1.07 \mathrm{~g}(90 \%)$.

PAUSE POINT Product 6 can be stored safely at $4{ }^{\circ} \mathrm{C}$ for $3-4$ weeks.

\section{Synthesis of 3-ethynyl-2,2,5,5-tetramethyl-1-oxy-pyrroline (7) $\bigcirc$ TIMING $1 \mathrm{~d}$}

30| Add a 20-ml THF solution of $1.17 \mathrm{~g}(11.6 \mathrm{mmol})$ diisopropylamine dropwise to a 50-ml round-bottom flask equipped with a magnetic stir bar, and then cool the reaction mixture to $0{ }^{\circ} \mathrm{C}$ by placing the reaction flask in an ice-water bath.

31 Add, dropwise, $7.23 \mathrm{ml}$ of a $1.6 \mathrm{M}$ hexane solution $(11.6 \mathrm{mmol})$ of $n$-butyllithium to the reaction mixture under a nitrogen atmosphere and continue stirring for $25 \mathrm{~min}$.

32 Cool the reaction mixture to $-78^{\circ} \mathrm{C}$ by placing the flask containing it in an acetone-dry ice bath and gradually add, over $5 \mathrm{~min}, 1.0 \mathrm{~g}(4.98 \mathrm{mmol})$ of product 6 from Step 29. Gradually warm the reaction mixture to room temperature over a period of $2 \mathrm{~h}$.

33| Quench the reaction by adding to the reaction mixture $2 \mathrm{ml}$ of ice-cold water. To the resulting mixture, add $10 \mathrm{ml}$ of water and extract with $\mathrm{Et}_{2} \mathrm{O}(3 \times 10 \mathrm{ml})$. Combine the organic layers and dry them over anhydrous $\mathrm{Na}_{2} \mathrm{SO}_{4}$. After stirring the mixture for a few minutes, filter the solid $\mathrm{Na}_{2} \mathrm{SO}_{4}$ and concentrate it in vacuo. Purify the crude product over a silica gel column $(\sim 3 \mathrm{~cm}$ internal diameter $\times 25 \mathrm{~cm}$ length) using EtOAc:hexane $(10: 90$, vol/vol) as the eluant to isolate compound 7 as a bright yellow solid. Yield: $642 \mathrm{mg}(78.5 \%)$.

PAUSE POINT Product 7 can be stored safely at $4{ }^{\circ} \mathrm{C}$ for $5-6$ months.

Synthesis of 5-(3-ethynyl-2,2,5,5-tetramethyl-1-oxy-pyrroline)-2'-deoxyuridine (8) $\bigcirc$ TIMING $1.5 \mathrm{~d}$

$34 \mid$ To a 100 -ml round-bottom flask equipped with a stir bar, add $0.50 \mathrm{~g}(3.04 \mathrm{mmol})$ of compound 7 (from Step 33$), 1.08 \mathrm{~g}$ $(3.04 \mathrm{mmol})$ of 5-iodo-2'-deoxyuridine, $114 \mathrm{mg}(0.60 \mathrm{mmol})$ of CuI and $346 \mathrm{mg}(0.30 \mathrm{mmol})$ of $\mathrm{Pd}\left(\mathrm{PPh}_{3}\right)_{4}$.

35| Dry the mixture for $30 \mathrm{~min}$ in vacuo. Seal the reaction vessel with a rubber septum and add $10 \mathrm{~mL}$ of anhydrous DMF to it with a syringe.

36| Deoxygenate the reaction mixture by alternately creating vacuum in the overhead space of the reaction flask and purging it with argon; repeat this procedure four or five times. Cover the flask with aluminum foil after deoxygenation in order to avoid exposure of the reaction mixture to light.

37| Add, dropwise, anhydrous $\mathrm{Et}_{3} \mathrm{~N}(1.0 \mathrm{ml}, 7.6 \mathrm{mmol})$ with a syringe and stir the reaction mixture overnight at room temperature.

38 Evaporate the reaction mixture in vacuo. Purify the crude product over a silica gel column $(\sim 3 \mathrm{~cm}$ internal diameter $x$ $25 \mathrm{~cm}$ length) using 3-5\% ( $\mathrm{vol} / \mathrm{vol}$ ) methanol in DCM as the eluant to isolate compound 8 as a light brown solid.

Yield: $975 \mathrm{mg}(82 \%)$.

- PAUSE POINT Product 8 can be stored safely at $4{ }^{\circ} \mathrm{C}$ for $8-10$ months.

Synthesis of 5-(3-ethynyl-2,2,5,5-tetramethyl-1-oxy-pyrroline)-5'-0-(4,4'-dimethoxytrityl)-2'-deoxyuridine (9) TIMING $1.5 \mathrm{~d}$

39 | Add $0.5 \mathrm{~g}(1.28 \mathrm{mmol})$ of compound 8 into a $100-\mathrm{ml}$ round-bottom flask, and then add $10 \mathrm{ml}$ of anhydrous pyridine. Evaporate all the volatiles using a rotary evaporator at $30^{\circ} \mathrm{C}$. Repeat this procedure 2 or 3 times. Add to the flask a magnetic stir bar and $20 \mathrm{ml}$ of anhydrous pyridine.

40| Cover the reaction flask with a $\mathrm{CaCl}_{2}$ guard tube to prevent atmospheric moisture from seeping in.

41| Add DMT-Cl (867 mg, $2.56 \mathrm{mmol})$ and a catalytic amount of DMAP.

42 Continue stirring overnight at room temperature. Remove the reaction volatiles in vacuo and purify the residue over a silica gel column $\left(\sim 3 \mathrm{~cm}\right.$ internal diameter $\times 15 \mathrm{~cm}$ length), first use as eluant hexane:EtOAc, 70:30 $+1 \%(\mathrm{vol} / \mathrm{vol}) \mathrm{Et}_{3} \mathrm{~N}$, 
then $\mathrm{EtOAc}+1 \% \mathrm{Et}_{3} \mathrm{~N}$ and finally elute product 9 with 1-2\% (vol/vol) methanol in DCM (with $1 \% \mathrm{Et}_{3} \mathrm{~N}$ ) as a light yellow solid. Yield: $816 \mathrm{mg}(92 \%)$.

PAUSE POINT Product 9 can be stored safely at $4{ }^{\circ} \mathrm{C}$ under an argon atmosphere for 8-9 months.

Synthesis of 5-(3-ethynyl-2,2,5,5-tetramethyl-1-oxy-pyrroline)-5'-0-(4,4'-dimethoxytrityl)-2'-deoxyuridine-3'(2-cyanoethyl-N,N-diisopropyl)phosphoramidite (10) $\bigcirc$ TIMING $1 \mathrm{~d}$

43| Place $150 \mathrm{mg}(0.216 \mathrm{mmol})$ of compound 9, from Step 42, in a 50-ml round-bottom flask equipped with a magnetic stir bar. Seal the reaction flask with a rubber septum and purge the overhead space with argon.

44| Dissolve the compound in $10 \mathrm{ml}$ of anhydrous DCM and then deoxygenate the resulting solution by creating vacuum in the overhead space of the reaction flask and purging it with argon; repeat this procedure 3 or 4 times.

45| Cool the reaction mixture by placing the reaction vessel in an ice bath. Add $191 \mu \mathrm{l}(1.08 \mathrm{mmol})$ of DIPEA dropwise with a syringe and stir the reaction mixture for $10 \mathrm{~min}$ at ${ }^{\circ} \mathrm{C}$.

46 Gradually add $102 \mathrm{mg}(0.433 \mathrm{mmol})$ of 2-cyanoethyl $\mathrm{N}, \mathrm{N}$-diisopropylchlorophosphoramidite with a syringe and continue stirring for $30 \mathrm{~min}$ at $0^{\circ} \mathrm{C}$.

47| Evaporate the reaction volatiles using a rotary evaporator at room temperature and purify the crude product over a silica gel column $\left(\sim 1.5 \mathrm{~cm}\right.$ internal diameter $\times 8 \mathrm{~cm}$ length). Elute the product with hexane:Et0Ac; 40:60 $+1 \%(\mathrm{vol} / \mathrm{vol}) \mathrm{Et}_{3} \mathrm{~N}$, under a continuous stream of nitrogen (nitrogen pressure was used during column chromatography instead of regular air pressure) to isolate product 10 as an off-white solid featuring an EPR spectrum, as shown in Figure 5, obtained by a routine continuous wave EPR experiment. Yield: $172 \mathrm{mg}(89 \%)$.

$\triangle$ CRITICAL STEP Store product 10 at $-20^{\circ} \mathrm{C}$ in a dry flask under an argon atmosphere and avoid air exposure; otherwise, phosphoramidite product will oxidize from phosphorus (III) to the phosphorus (V) species.

PAUSE POINT Product 10 can be stored safely at $-80^{\circ} \mathrm{C}$ under an argon atmosphere for 4-6 months.

\section{DNA synthesis TIMING variable, depending on oligonucleotide length}

48| For each coupling reaction, dissolve the synthesized TPA spin-labeled phosphoramidite (compound 10) in $\sim 150 \mu \mathrm{l}$ of anhydrous acetonitrile to a final concentration of $0.1 \mathrm{M}$. Place the vial with the spin-labeled phosphoramidite in a free position of the synthesizer. As the sample is being placed in the synthesizer, flush the vial with argon.

49| Enter in the synthesizer's computer commands in order to synthesize the desired DNA sequence. Install the CPG column with the appropriate first 3' base attached. Perform the synthesis under standard conditions as described in the synthesizer manual. Use a coupling time of 15 min with several short pushes to incorporate the TPA-labeled phosphoramidite into the nascent oligonucleotide. The usual coupling time for nonlabeled phosphoramidites is $30 \mathrm{~s}$. Prepare the oligonucleotides with the 'DMT on' mode.

$\triangle$ CRITICAL STEP The nitroxide radical is reduced to the corresponding (EPR inactive) amine in acidic conditions (deblocking-TCA reagent). To minimize this reduction, synthesize the oligonucleotides with trityl monitoring off. ? TROUBLESHOOTING

50| Remove the column from the synthesizer, dry it carefully in vacuo and store it in an argon gas atmosphere; it can be stored for several weeks at $4^{\circ} \mathrm{C}$.

\section{DNA purification TIMING $4 \mathrm{~d}$}

51| Cleave the spin-labeled oligonucleotides from the CPG material and remove nucleobase-protecting groups. For this purpose, transfer the CPG beads from the column into a 2-mL screw-cap vial and add to it $1 \mathrm{ml}$ of a $28-30 \%$ (wt/wt) ammonium hydroxide solution. Incubate the mixture at $55{ }^{\circ} \mathrm{C}$ for at least $10 \mathrm{~h}$.

52 Evaporate excess ammonia by keeping the vial in a vacuum concentrator for $1 \mathrm{~h}$ at $45^{\circ} \mathrm{C}$.

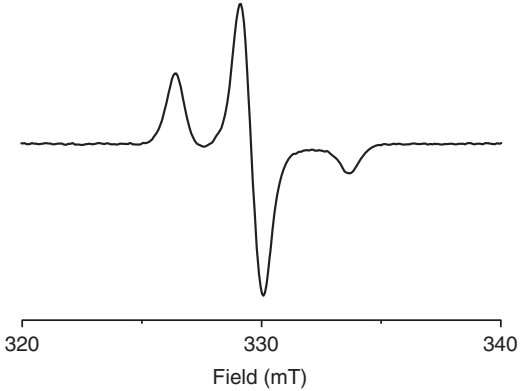

Figure 5 | CW-EPR spectrum of TPA-labeled phosphoramidite. $T=120 \mathrm{~K}$, $X$ band. 
Figure 6 | HPLC purification of spin-labeled oligonucleotides. Chromatogram of the sequence 5'-GCCGIATCGAAGCGC. The TPA-modified nucleotide is bold and underlined. (a) 'DMT on' chromatogram. (b) 'DMT off' chromatogram. The collected fractions are marked with black arrows.

53| Separate the beads from the oligonucleotides by filtering the contents of the vial from Step 52 through a 0.2- $\mu \mathrm{m}$ filter, and then wash the beads left on the filter twice with Millipore water. a

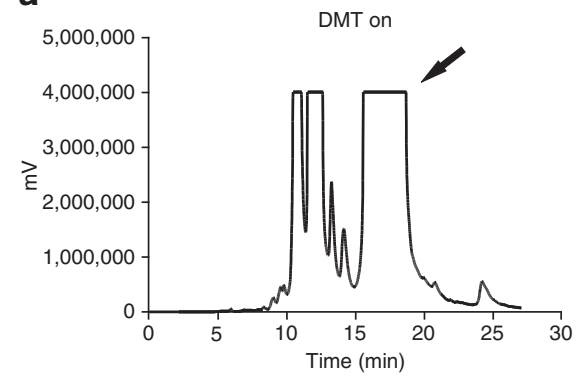

b

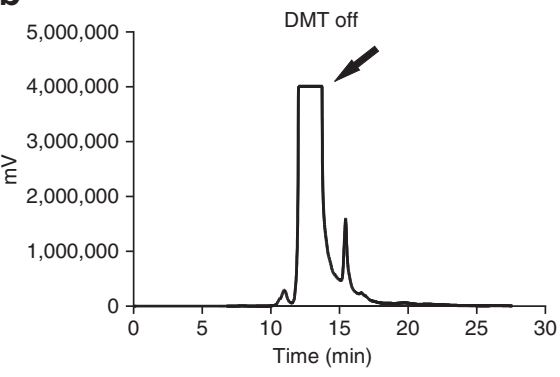

54| Purify the DNA in the filtrate via reverse-phase HPLC by using the 'DMT on' mode. Use 0.1 M TEAA and acetonitrile as solvents and apply a gradient from $95 \% / 5 \%$ to $30 \% / 70 \%$ over a period of $30 \mathrm{~min}$. Under these conditions, the DMTprotected DNA usually elutes after 15-20 min. Side products, such as shorter oligonucleotides, elute earlier from the column than the expected oligonucleotides. Please note that the elution time of DMT-protected oligonucleotides depends on the gradient you apply and the type of column you use. A typical chromatogram is shown in Figure 6a. After collecting the HPLC fractions containing the desired oligonucleotide, evaporate the solvent in a SpeedVac at room temperature.

55| Re-dissolve the DNA in 400-500 $\mu$ l of Millipore water and store it for at least $2 \mathrm{~d}$ at $4{ }^{\circ} \mathrm{C}$. During this time, almost quantitative cleavage of the DMT-protective group takes place; the occurrence of this cleavage can be confirmed by performing a second HPLC purification using the 'DMT-off' mode; see Step 56.

$\triangle$ CRITICAL STEP Do not use trichloroacetic acid for DMT deprotection at this point because this compound reduces the nitroxide spin label ${ }^{12}$.

56| Perform a second HPLC purification on the DNA solution of Step 55 using the 'DMT-off' mode. Again use 0.1 M TEAA/ acetonitrile as the eluant and a gradient from $95 \% / 5 \%$ to $30 \% / 70 \%$ over $30 \mathrm{~min}$. The oligonucleotide without the DMTprotecting group usually elutes after 12-15 min. There might be a residual fraction of the oligonucleotide that is still DMT protected. This fraction is, however, more hydrophobic than the 'DMT-less' fraction and therefore elutes later in reverse-phase HPLC. A typical chromatogram is shown in Figure $6 \mathrm{~b}$.

57| After collecting the desired HPLC fractions, vaporize the solvents using a SpeedVac at room temperature. Note that TEAA is usually not fully removed by a single vaporization step, and thus the next step completes the removal of TEAA.

58| Dissolve the DNA in Millipore water again $(\sim 400 \mu \mathrm{l})$, and lyophilize the DNA to remove solvent residues and leftover TEAA. Analyze it by ESI mass spectrometry.

? TROUBLESHOOTING

\section{Surgical excision of stage VI oocytes TIMING 2 h}

59| If you are not using preharvested $X$. laevis ovaries from a commercial vendor, proceed with Step 60 of the procedure; alternatively, go to Step 67.

60 0 To induce anesthesia in a 3- to 5-year-old female $X$. laevis placed in a small holding tank, add ethyl 3-aminobenzoate to the tank containing water to obtain a final concentration of $0.1 \%$ (wt/vol) ethyl 3-aminobenzoate. Anesthesia will occur within 5-7 min. Once anesthesia has set in, place the female Xenopus onto shaved ice in order to maintain anesthesia and retain immobilization. To avoid freeze burns and dehydration, place the frog on a wetted paper towel on the shaved ice. In addition, cover the whole frog with wetted sterile paper towels with the exception of the surgical area.

I CAUTION Proper animal care and safety protocols must be adhered to at all times.

61| Open the abdominal area (skin and muscle layer) with a scalpel, gently tease parts of the ovary outside of the body and open the ovary bags.

62 Cut the oocyte strings from the ovary bags and immediately transfer the oocyte strings into a 50-ml conical centrifuge tube containing $40 \mathrm{ml}$ of $1 \times$ MBS medium at $18^{\circ} \mathrm{C}$. 


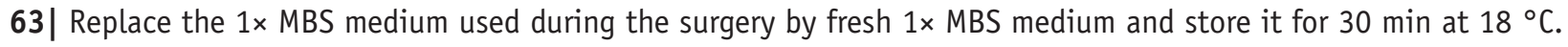

64| Transfer oocyte strings to a plastic Petri dish containing $30 \mathrm{ml}$ of defolliculation medium, and then open the oocyte strings with forceps and gently shake the Petri dish with the rough detached oocyte strings for $2 \mathrm{~h}$ at $18{ }^{\circ} \mathrm{C}$ to free the oocytes.

65 Wash the oocytes three times with $1 \times$ MBS medium, and then select and collect stage VI oocytes. Stage VI cells can be easily identified, as they are the largest oocytes in the population. Store the oocytes for $4-24 \mathrm{~h}$ at $18{ }^{\circ} \mathrm{C}$.

\section{0ocyte microinjection and EPR sample preparation $\bigcirc$ TIMING $2 \mathrm{~d}$}

66| Manually sort out healthy-looking stage VI oocytes. Typically, stage VI cells are healthy when the darkly pigmented hemisphere (the animal pole) appears uniformly colored and with a sharp border to the vegetal pole (Fig. 7).

67| Prepare the microinjector. Fill a pulled injection capillary tip (glass, 8 inches) with oil and set it up to the Drummond Digital Microdispenser 500 system; slightly break the capillary and collect the sample for injection. Place the immobilized oocyte under a dissection microscope and penetrate the animal hemisphere of the cell with the injection needle.

$\Delta$ CRITICAL STEP Caution should be taken to insert the needle smoothly and with as little friction as possible, so as to avoid damaging the cell.

68| After injecting the sample in the oocyte, withdraw the needle from the oocyte with the same precision and care used to insert it.

69| Inspect the manipulated cell by focusing on the injection point and sort out any obviously damaged cells (cells leaking fluid or proteins). Inject the desired number of cells by repeating Steps 66-68.

70| Incubate the oocytes at room temperature for a chosen length of time, taking into account the lifetime of the spin label and the expected kinetics of oligonucleotide folding (consult refs. 6, 8 and 12 for detailed discussions on this subject). A period of several minutes is a reasonable starting incubation time.

71 After incubation, transfer the injected oocyte with a Pasteur pipette to an Eppendorf tube and place the tube in liquid nitrogen to shock-freeze the oligonucleotides in their equilibrium conformations.

! CAUTION Wear protective goggles and gloves while working with liquid nitrogen.

$\triangle$ CRITICAL STEP To avoid changes in the trapped DNA conformations, the oocytes must be kept frozen for all subsequent steps.

72| Place the lyophilizer on dry ice. Place the frozen oocytes in the lyophilizer in order to remove the ice cover that has been deposited on the surface of the oocytes. Keep the oocytes in the lyophilizer until the ice cover is removed, which usually takes $24-48 \mathrm{~h}$.

$\Delta$ CRITICAL STEP Check the appearance of the oocytes regularly. When the ice cover has been removed, proceed immediately with Step 73 to avoid dehydration of the cells.

73| Equip the EPR tube with a funnel fashioned from a sheet of weighing paper. Place the EPR tube in dry ice.

74| Insert the oocytes carefully into the EPR tube and allow them to sediment by gravity. Swirl the tube to ensure optimal packing of the oocytes (Fig. 2).

$\triangle$ CRITICAL STEP Ensure that the oocytes are kept frozen throughout this delicate step.

75| Count and take note of the number of oocytes per EPR sample, as recording this number is crucial to enabling researchers to correlate the effective concentration of the sample to the intracellular molarity of the DNA sequence being investigated.

! CAUTION Wear protective goggles and gloves while working with items stored at $-80^{\circ} \mathrm{C}$.

- PAUSE POINT The EPR sample can be immediately used for the measurement or can be stored at $-80^{\circ} \mathrm{C}$ for several weeks before use.
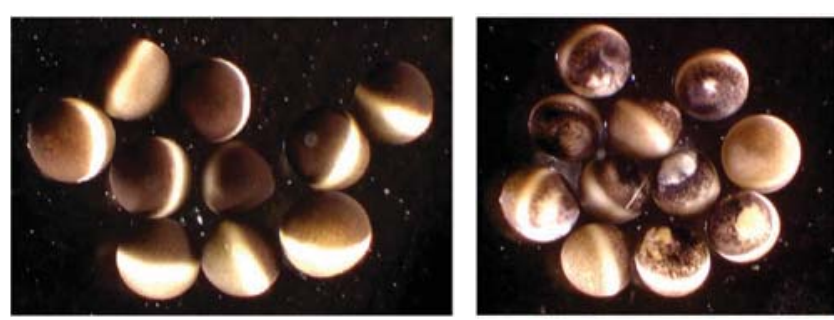

Figure 7 | Optical images of stage VI $X$. laevis oocytes. Healthy (left) and necrotic (right) oocytes. 


\section{In-cell EPR measurement $\bigcirc$ TIMING $24 \mathrm{~h}$}

76| Switch on the EPR spectrometer according to the user manual.

77| Cool down the cryostat to $120 \mathrm{~K}$.

78| Couple the resonator critically according to the user manual.

79| Mount the EPR tube containing the frozen sample of oocytes in the sample holder or sample rod assembly kept in liquid nitrogen.

$80 \mid$ Turn the helium pump off. Ensure that the needle valve on the flow controller is not closed.

81| Monitor the pressure gauge on the flow controller. Wait for at least $10 \mathrm{~s}$ after the gauge indicates $1 \mathrm{~atm}$ of pressure.

82| Switch the EPR spectrometer to standby.

83 Quickly remove the empty sample rod or the EPR tube (with the sample just analyzed) from the resonator.

! CAUTION Wear safety glasses. Avoid contact with the cold objects. Be extremely careful when you handle samples that are rapidly heated or cooled. The rapid cooling of some samples may result in the formation of a solid bolus in the EPR tube that may make the tube prone to explosive rupture.

84| Pick up the EPR tube mounted in the sample holder or sample rod assembly in Step 79. Pull the stopper out of the top of the sample rod and insert the frozen sample. Take $\sim 5 \mathrm{~s}$ to fully insert the sample and then reinsert the stopper. If inserting the sample in the resonator takes more than $5 \mathrm{~s}$, insert an empty sample rod into the resonator to prevent air from entering it.

$\triangle$ CRITICAL STEP Ensure that the EPR tube is placed in the optimum position as described in the resonator manual.

85| Switch the EPR spectrometer to standby.

86| Turn the helium pump on. Wait for 15 min for your sample to come to thermal equilibrium.

87| Switch the EPR spectrometer to operate.

88| Adjust the resonant microwave frequency to the center of the resonator frequency.

89| Switch the spectrometer to CW mode. Set the microwave attenuation to $60 \mathrm{~dB}$. Adjust the diode current to $200 \mu \mathrm{A}$ via the bias slider.

90| Decrease the attenuation to $30 \mathrm{~dB}$ and adjust both coupling and frequency to obtain a diode current of $200 \mu \mathrm{A}$ and a lock offset value of 0 . Decrease attenuation in sequential steps of $1 \mathrm{~dB}$ down to $10 \mathrm{~dB}$, in order to check whether both diode current and the lock offset remain the same. Correct both coupling and frequency if necessary.

91 Acquire a CW-EPR spectrum using appropriate experimental parameters, such as 3355 Gauss center field, frequency modulation $100 \mathrm{kHz}$, modulation amplitude $1.0 \mathrm{G}$, power $8 \mathrm{~mW}$, time constant $20.48 \mathrm{~ms}$, conversion $40.96 \mathrm{~ms}, 1,024$ points, sweep time $42 \mathrm{~s}$ and sweep width $110 \mathrm{G}$. Also run an appropriate number of scans to yield a reasonable signal-to-noise ratio. This number can range from 10 to 300 , depending on the spectral width.

? TROUBLESHOOTING

92 Cool the cryostat down to a working temperature of $45 \mathrm{~K}$. Completely overcouple the resonator. Readjust the resonant microwave frequency to the center of the resonator if necessary (the resonance frequency may shift upon cooling down from 120 to $45 \mathrm{~K}$ ). This is the pump frequency (Fig. 3c).

93 Switch the spectrometer to pulse mode. Set the microwave attenuation to $60 \mathrm{~dB}$. Switch the traveling wave tube amplifier to operate for amplification of the microwave pulses. Apply a pulse sequence containing one pulse of $16 \mathrm{~ns}$ of length. Slowly decrease the attenuation to $0 \mathrm{~dB}$. 
$\triangle$ CRITICAL STEP Check that no ringing can be detected from the resonator when applying the pulse with $0 \mathrm{~dB}$ (full power). Otherwise, the detection electronics may be destroyed.

? TROUBLESHOOTING

94 Apply the Hahn-echo sequence $\pi / 2-\tau-\pi-\tau-$ echo $(\pi / 2$ and $\pi$ pulses are 16 and 32 ns long, respectively, and $\tau=200 \mathrm{~ns})$. Set the shot repetition time to $4 \mathrm{~ms}$ and the video amplifier bandwidth to $20 \mathrm{MHz}$.

95| Adjust the echo intensity. Find appropriate microwave attenuation and magnetic field to maximize the echo (Fig. $3 \mathbf{b}$ ). The starting value for the magnetic field can be estimated from the center of the CW spectrum recorded in Step 91. Adjust the phase to maximum positive echo intensity in the real part of the signal. Increase the repetition rate until the echo intensity starts decreasing.

? TROUBLESHOOTING

96| To adjust the 24-ns pump pulse at this microwave frequency (pump frequency), use the inversion recovery pulse sequence $\pi-\tau_{1}-\pi / 2-\tau_{2}-\pi-\tau_{2}-$ echo. The first $\pi$ pulse is applied via the ELDOR channel; its frequency is set to the pump frequency. This pulse is $24 \mathrm{~ns}$ long and placed $400 \mathrm{~ns}$ before the Hahn-echo sequence (see Step 89). Optimize the ELDOR attenuation to have maximum negative echo amplitude corresponding to inversion.

? TROUBLESHOOTING

97| Set the operating frequency $67 \mathrm{MHz}$ higher than the pump frequency (Fig. 3b). This value is defined as the observer frequency. Apply the Hahn-echo sequence $\pi / 2-\tau-\pi-\tau-$ echo $(\pi / 2$ and $\pi$ pulses are 16 and 32 ns long, respectively, and $\tau=200 \mathrm{~ns}$ ) for $+<x>$ and $-<x>$ channels. Maximize the echo intensity by varying the attenuation. Adjust the phases so as to observe a phase shift of $180^{\circ}$ between the two pulse channels.

98 Apply the $\pi / 2-\tau_{1}-\pi-\tau_{1}-$ echo $-\tau_{2}-\pi-\tau_{2}-$ refocused echo sequence at the observer frequency $\left(\tau_{1}=200 \mathrm{~ns}\right.$, $\tau_{2}$ initial value $\left.=1 \mu \mathrm{s}\right)$. Set the SpecJet scale factor to one. Adjust $\tau_{2}$ by inspecting the refocused echo. Set $\tau_{2}$ to the longest value at which the refocused echo can still be clearly recognized. Typical values are between 1 and $3.5 \mu$ s.

99| Apply the DEER sequence (Fig. 3a). The pump inversion pulse (at the pump frequency on resonance with the microwave cavity) with a length of 24 ns and an amplitude determined in Step 96 starts with a delay time $<2 \tau_{1}$ but is large enough not to overlap with the first observer $\pi$-pulse. The pump pulse is swept within the experiment in $8 \mathrm{~ns}$ steps, resulting in the DEER trace.

100| Set the phase cycle for the observer sequence to be $\pi / 2(+<x>)-\pi(<y>)-\pi(<y>)$ and $\pi / 2(-<x>)-\pi(<y>)-$ $\pi(<y>)$.

? TROUBLESHOOTING

101| Apply nuclear modulation suppression by averaging DEER traces for ten different $\tau_{1}$ values incremented by 8 ns (ref. 34).

? TROUBLESHOOTING

102| Set the integration window (length: 32 ns) symmetrically over the refocused echo.

103 Set the number of averages for the SpecJet to 1. Adjust the gain so that the signal intensity does not exceed the SpecJet window.

104| Increase the number of scans and shots per point to repeat the experiment as often as required in order to obtain an acceptable signal-to-noise ratio. Depending on spectrometer stability, one should not exceed accumulation times of $24 \mathrm{~h}$.

105| Save data for the following data analysis. A sketch of the data analysis is provided in Figure 4.

106| If data on another sample need to be acquired, repeat Steps 77-105.

\section{Data postprocessing and analysis by DeerAnalysis TIMING variable}

107| If EPR data on a singly spin-labeled sample of oocyte-injected oligonucleotide solution are available, go to Step 108; otherwise, go to Step 117. 
108 Load the data for the singly spin-labeled sample into the DeerAnalysis software. One detection channel is referred to as the real part, and the second as the imaginary part. Set the correct value for the zero time as determined according to the DeerAnalysis manual.

109| Check for proper phase correction: the signal should be in the real part, and the imaginary part should be approximately zero; otherwise, manually correct the phase.

110| Cut data as suggested by the DeerAnalysis software.

111 Set the background start at 0 ns. Fit the trace with a polynomial function. Select the polynomial with the lowest order that still gives a good fit. Save the polynomial function selected.

112 Load the data for the doubly spin-labeled sample into the DeerAnalysis software. Set the zero time to the same value as in Step 108.

113| Check for proper phase correction; see Step 109.

$114 \mid$ Cut data as suggested by DeerAnalysis.

115 Set the background start as suggested by DeerAnalysis.

116 Perform the background correction on the data on the doubly spin-labeled sample using the polynomial derived in Step 111.

? TROUBLESHOOTING

117| Load the data for the doubly spin-labeled sample into the DeerAnalysis software. Set the correct value for the zero time according to Step 108.

118| Check for proper phase correction: the signal should be in the real part and the imaginary part should be approximately zero; otherwise, manually correct the phase.

$119 \mid$ Cut data as suggested by the DeerAnalysis software.

120| Perform the background correction using the homogeneous background function.

? TROUBLESHOOTING

121 Determine the optimum regularization parameter using the ' $\mathrm{L}$ curve' tool of the DeerAnalysis software according to the manual.

122 Extract the distance distribution via Tikhonov regularization using the DeerAnalysis software and the regularization parameter determined in Step 121.

? TROUBLESHOOTING

123| Perform a statistical analysis of the distance distribution thus obtained using the relevant tool in DeerAnalysis. To achieve this objective, follow the suggestion in the DeerAnalysis user manual.

\section{? TROUBLESHOOTING}

Troubleshooting advice can be found in Table 1. 
TABLE 1 | Troubleshooting table.

\begin{tabular}{lll}
\hline Step Problem & Possible reason & Solution
\end{tabular}

Synthesis of spin-labeled oligonucleotides

49 DNA synthesis does not yield The reagents are decomposed sufficient amounts of product

The phosphoramidites are lost

The tubes in the synthesizer are blocked

The pressure is not appropriate

\section{DNA purification}

58 The oligonucleotide does not Spin-labeled nucleotide is impure have the expected mass

Deprotection did not work

\section{In-cell DEER measurement}

91 The EPR intensity of the CW signal is too low
93

$95 \quad$ No echo
No inversion of the echo by the pump pulse is observed

100 The signal averages to zero after phase cycling

101 Proton modulations are very strong

116, The DEER curve shows a

120 monoexponential decay only

122 Distance distribution is spread over the whole accessible range of distances
The chosen incubation time was too long

The position of the sample in the resonator is incorrect

Ice cover was not properly removed from oocytes so that the most dense packing in the EPR tube was not achieved

0ocytes were allowed to thaw in the time it took to place them in the spectrometer

Resonator coupling is too strong or the protection pulse is too short

Incorrect magnetic field setting

The overcoupling of the resonator is too strong

The phases at $+<x>$ and $-<x>$ channels are set incorrectly

May be a characteristic of the particular sample

The degree of labeling is insufficient

The oocytes were in the lyophilizer too long and the cells became dehydrated
Replace all the reagents

Replace the phosphoramidites or check the purity with a 31P-NMR

Flush all the tubes with acetonitrile

Control the pressure in the synthesizer

Purify the spin-labeled DNA via HPLC

Leave the sample in the refrigerator for $2 \mathrm{~d}$ and purify again

Reduce the incubation time

Check the sample positioning

Remove oocytes from the lyophilizer only after the ice cover disappears

Ensure that oocytes remain frozen during all transfer procedures

Fully decouple the resonator; increase the length of the protection pulse

Detect the echo at the magnetic field that corresponds to the maximum of the nitroxide spectrum (estimated from CW measurement in Step 91)

Reduce the coupling but check for ringing and check the resonator width

Recheck the phases adjusted for the observer frequency

Filter during data postprocessing

Increase the extent of labeling and/or reduce the incubation time

Carefully observe oocytes in the lyophilizer and remove them as soon as the ice cover disappears

\section{TIMING}

Steps 1-4, synthesis of 3,5-dibromo-2,2,6,6-tetramethylpiperidin-4-one hydrobromide (2): $2 \mathrm{~d}$ Steps 5-12, synthesis of 3-(N-methoxy- $N$-methylcarbamoyl)-2,2,5,5-tetramethylpyrroline (3): $2 \mathrm{~d}$ Steps 13-17, synthesis of 3-(N-methoxy- $N$-methylcarbamoyl)-2,2,5,5-tetramethyl-1-oxy-pyrroline (4): $1 \mathrm{~d}$ 
Steps 18-22, synthesis of 3-formyl-2,2,5,5-tetramethyl-1-oxy-pyrroline (5): $1 \mathrm{~d}$

Steps 23-29, synthesis of 3-(2-chloroethyl)-2,2,5,5-tetramethyl-1-oxy-pyrroline (6): $1 \mathrm{~d}$

Steps 30-33, synthesis of 3-ethynyl-2,2,5,5-tetramethyl-1-oxy-pyrroline (7): $1 \mathrm{~d}$

Steps 34-38, synthesis of 5-(3-ethynyl-2,2,5,5-tetramethyl-1-oxy-pyrroline)-2'-deoxyuridine (8): $1.5 \mathrm{~d}$

Steps 39-42, synthesis of 5-(3-ethynyl-2,2,5,5-tetramethyl-1-oxy-pyrroline)-5'-0-(4,4'-dimethoxytrityl)-2'-deoxyuridine (9): $1.5 \mathrm{~d}$ Steps 43-47, synthesis of 5-(3-ethynyl-2,2,5,5-tetramethyl-1-oxy-pyrroline)-5'-0-(4,4'-dimethoxytrityl)-2'-deoxyuridine-3'(2-cyanoethyl- $N, N$-diisopropyl)phosphoramidite (10): $1 \mathrm{~d}$

Steps 48-50, DNA synthesis: variable, depending on the oligonucleotide length; cycle times: $\sim 5$ min per incorporation of standard deoxynucleotides, 20 min per spin-labeled nucleotide

Steps 51-58, DNA purification: $4 \mathrm{~d}$

Steps 59-65, surgical excision of stage VI oocytes: $2 \mathrm{~h}$

Steps 66-75, oocyte microinjection and preparation of EPR samples: $\sim 2 \mathrm{~d}$

Steps 76-106, in-cell DEER measurement: $24 \mathrm{~h}$

Steps 107-123, data postprocessing: variable; from 30 min to several days

\section{ANTICIPATED RESULTS}

\section{Analytical data}

3,5-Dibromo-2,2,6,6-tetramethylpiperidin-4-one hydrobromide (2). ${ }^{1} \mathrm{HNMR}\left(\mathrm{CD}_{3} \mathrm{OD}, 250 \mathrm{MHz}, \delta\right.$ p.p.m.): 5.68 (s, $2 \mathrm{H}, 2 \times$ $\mathrm{CHBr}), 1.89\left(\mathrm{~s}, 6 \mathrm{H}, 2 \times \mathrm{CH}_{3}\right), 1.45\left(\mathrm{~s}, 6 \mathrm{H}, 2 \times \mathrm{CH}_{3}\right)$.

3-(N-methoxy- $\mathrm{N}$-methylcarbamoyl)-2,2,5,5-tetramethylpyrrolin (3). ${ }^{1 \mathrm{HNMR}}\left(\mathrm{CDCl}_{3}, 250 \mathrm{MHz}, \delta\right.$ p.p.m.): 6.03 (s, $\left.1 \mathrm{H},=\mathrm{CH}\right)$, $3.54\left(\mathrm{~s}, 3 \mathrm{H}, 0 \mathrm{CH}_{3}\right), 3.15\left(\mathrm{~s}, 3 \mathrm{H}, \mathrm{NCH}_{3}\right), 1.32\left(\mathrm{~s}, 6 \mathrm{H}, 2 \times \mathrm{CH}_{3}\right), 1.21\left(\mathrm{~s}, 6 \mathrm{H}, 2 \times \mathrm{CH}_{3}\right)$.

3-(N-methoxy- $N$-methylcarbamoyl)-2,2,5,5-tetramethyl-1-oxy-pyrrolin (4). Please note that for this compound, and for all other compounds containing a paramagnetic moiety (compounds 4-10), NMR spectroscopy is not useful as an analytical tool. To characterize this type of molecule by NMR, the paramagnetism needs to be quenched beforehand. For that purpose (nondeuterated), phenylhydrazine can be added to the NMR solution of the compound at hand and the NMR spectrum collected. The phenylhydrazine peaks are then subtracted from the observed spectrum. On applying the approach just described, the NMR analytical data of spin-quenched 4 are as follows: ${ }^{1} \mathrm{HNMR}\left(\mathrm{CDCl}_{3}+\right.$ phenylhydrazine, $400 \mathrm{MHz}, \delta$ p.p.m.): $6.10(\mathrm{~s}, 1 \mathrm{H},=\mathrm{CH}), 3.56\left(\mathrm{~s}, 3 \mathrm{H}, 0 \mathrm{CH}_{3}\right), 3.16\left(\mathrm{~s}, 3 \mathrm{H}, \mathrm{NCH}_{3}\right), 1.41\left(\mathrm{~s}, 6 \mathrm{H}, 2 \times \mathrm{CH}_{3}\right), 1.31\left(\mathrm{~s}, 6 \mathrm{H}, 2 \times \mathrm{CH}_{3}\right)$.

3-Formyl-2,2,5,5-tetramethyl-1-oxy-pyrrolin (5) (spin-quenched). ${ }^{1} \mathrm{HNMR}\left(\mathrm{CDCl}_{3}+\right.$ phenylhydrazine, $400 \mathrm{MHz}, \delta$ p.p.m.): $9.53(\mathrm{CHO}), 6.46(\mathrm{~s}, 1 \mathrm{H},=\mathrm{CH}), 1.28\left(\mathrm{~s}, 6 \mathrm{H}, 2 \times \mathrm{CH}_{3}\right), 1.23\left(\mathrm{~s}, 6 \mathrm{H}, 2 \times \mathrm{CH}_{3}\right)$.

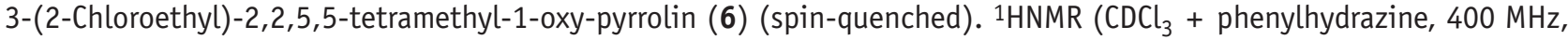
$\delta$ p.p.m.): 6.23-5.47 (m, $3 \mathrm{H}, 3 \times=\mathrm{CH}), 1.20\left(\mathrm{~s}, 6 \mathrm{H}, 2 \times \mathrm{CH}_{3}\right), 1.14\left(\mathrm{~s}, 6 \mathrm{H}, 2 \times \mathrm{CH}_{3}\right)$.

3-Ethynyl-2,2,5,5-tetramethyl-1-oxy-pyrrolin (7) (spin-quenched). ${ }^{1} \mathrm{HNMR}$ ( $\mathrm{CDCl}_{3}+$ phenylhydrazine, $\left.400 \mathrm{MHz}, \delta \mathrm{p} . \mathrm{p} . \mathrm{m}.\right)$ : $5.82(\mathrm{~s}, 1 \mathrm{H},=\mathrm{CH}), 2.90(\mathrm{~s}, 1 \mathrm{H}, \mathrm{CH}), 1.21\left(\mathrm{~s}, 6 \mathrm{H}, 2 \times \mathrm{CH}_{3}\right), 1.16\left(\mathrm{~s}, 6 \mathrm{H}, 2 \times \mathrm{CH}_{3}\right)$.

5-(3-Ethynyl-2,2,5,5-tetramethyl-1-oxy-pyrrolin)-2'-deoxyuridine (8) (spin-quenched). ${ }^{1} \mathrm{HNMR}\left(\mathrm{CD}_{3} \mathrm{OD}+\right.$ phenylhydrazine, $400 \mathrm{MHz}, \delta$ p.p.m.): $8.34(\mathrm{~s}, 1 \mathrm{H}, \mathrm{H} 6), 6.26\left(\mathrm{~m}, 1 \mathrm{H}, 1^{\prime} \mathrm{CH}\right), 5.91(\mathrm{~s}, 1 \mathrm{H},=\mathrm{CH}), 5.33-5.30\left(\mathrm{~m}, 1 \mathrm{H}, 4^{\prime} \mathrm{CH}\right), 4.11\left(\mathrm{~m}, 1 \mathrm{H}, 3^{\prime} \mathrm{CH}\right)$, $3.80\left(\mathrm{~m}, 2 \mathrm{H}, 5^{\prime} \mathrm{CH}_{2}\right), 2.44-2.23\left(\mathrm{~m}, 2 \mathrm{H}, 2^{\prime} \mathrm{CH}_{2}\right), 1.28\left(\mathrm{~s}, 6 \mathrm{H}, 2 \times \mathrm{CH}_{3}\right), 1.21\left(\mathrm{~s}, 6 \mathrm{H}, 2 \times \mathrm{CH}_{3}\right)$.

5-(3-Ethynyl-2,2,5,5-tetramethyl-1-oxy-pyrrolin)-5'-0-(4,4'-dimethoxytrityl)-2'-deoxyuridine (9) (spin-quenched). ${ }^{1} \mathrm{HNMR}$ $\left(\mathrm{CDCl}_{3}+\right.$ phenylhydrazine, $400 \mathrm{MHz}, \delta$ p.p.m. $): 8.80(\mathrm{~s}, 1 \mathrm{H}, \mathrm{H} 6), 7.61-6.90(\mathrm{~m} .13 \mathrm{H}, \mathrm{ArH}), 6.26-6.24\left(\mathrm{~m}, 1 \mathrm{H}, 1^{\prime} \mathrm{CH}\right)$, $5.92(\mathrm{~s}, 1 \mathrm{H},=\mathrm{CH}), 4.63-4.58\left(\mathrm{~m}, 1 \mathrm{H}, 4^{\prime} \mathrm{CH}\right), 4.04-4.03\left(\mathrm{~m}, 1 \mathrm{H}, 3^{\prime} \mathrm{CH}\right), 3.71\left(\mathrm{~s}, 6 \mathrm{H}, 2 \times 0 \mathrm{CH}_{3}\right), 3.56-3.42\left(\mathrm{~m}, 2 \mathrm{H}, 5^{\prime} \mathrm{CH}_{2}\right)$, 2.46-2.24 (m, 2H, 2' $\left.\mathrm{CH}_{2}\right), 1.22\left(\mathrm{~s}, 6 \mathrm{H}, 2 \times \mathrm{CH}_{3}\right), 1.20\left(\mathrm{~s}, 6 \mathrm{H}, 2 \times \mathrm{CH}_{3}\right)$.

5-(3-Ethynyl-2,2,5,5-tetramethyl-1-oxy-pyrrolin)-5'-0-(4,4'-dimethoxytrityl)-2'-deoxyuridine-3'-(2-cyanoethyl- $N, N$ diisopropyl)phosphoramidite (10) (spin-quenched). ${ }^{31} \mathrm{P} \mathrm{NMR}\left(\mathrm{CDCl}_{3}+\right.$ phenylhydrazine, $100 \mathrm{MHz}, \delta$ p.p.m.): $149.06,149.87$. Compound 10 features an EPR spectrum, as shown in Figure 5, in a routine continuous wave EPR experiment.

\section{Synthesis of spin-labeled DNA}

The typical yield of a purified, doubly TPA-spin-labeled DNA oligonucleotide $10 \mathrm{nt}$ long is $\sim 0.6 \mu \mathrm{mol}$ for a 1- $\mu$ mol synthesis scale. The synthesis scale refers to the amount of CPG support used to start the DNA synthesis. Please note that because of the presence of paramagnetic moieties in the compounds synthesized, NMR spectroscopy cannot be used to characterize the spin-labeled oligonucleotides; ESI-MS must be used instead.

\section{Microinjected oocytes}

Before Xenopus oocytes are injected with the solutions of spin-labeled oligonucleotides, their development stage (stage VI) can be reconfirmed using the morphological criteria described by Dumont ${ }^{35}$. Microinjected oocytes that display leakage or other visually discernible defects must be discarded, whereas healthy-looking oocytes (Fig. 7) are suitable for in-cell DEER experiments. 
In-cell DEER experiments

The modulation depth as defined in the DeerAnalysis manual should be at least $3-5 \%$ in order to calculate distance distribution ${ }^{36}$.

\section{DEER data processing}

Figure 4a shows a sketch of an ideal DEER curve (raw data, real part), which corresponds to the data used in Step 117. Upon background correction as performed in Step 116 or 120, respectively, one obtains the form factor depicted in Figure 4b. Figure 4c shows an example of a distance distribution as obtained in Step 122.

ACKNOWLEDGMENTS We thank M. Spitzbarth and M. Wassmer for preparing figures. Research in M.D.'s laboratory is supported by the Deutsche Forschungsgemeinschaft, the Ministry of Science, Research and the Arts of Baden-Württemberg and the German Excellence Initiative.

AUTHOR CONTRIBUTIONS M.A., J.S.H. and M.D. conceived the experimental strategy. J.S.H., D.R.D. and M.D. supervised the project. V.S. synthesized the labeled DNA sequences, 0.0. performed the microinjection and M.A. conducted the EPR experiments and data analysis. All authors contributed to the discussion and writing of the manuscript.

COMPETING FINANCIAL INTERESTS The authors declare no competing financial interests.

1. Pannier, M., Veit, S., Godt, A., Jeschke, G. \& Spiess, H.W. Dead-time free measurement of dipole-dipole interactions between electron spins. J. Magn. Reson. 142, 331-340 (2000).

2. Schiemann, 0 . et al. Spin labeling of oligonucleotides with the nitroxide TPA and use of PELDOR, a pulse EPR method, to measure intramolecular distances. Nat. Protoc. 2, 904-923 (2007).

3. Banham, J.E. et al. Distance measurements in the borderline region of applicability of $\mathrm{cW}$ EPR and DEER: a model study on a homologous series of spin-labelled peptides. J. Magn. Reson. 191, 202-218 (2007).

4. Pielak, G.J. \& Tian, F. Membrane proteins, magic-angle spinning, and incell NMR. Proc. Natl Acad. Sci. USA 109, 4715-4716 (2012)

5. Serber, Z. et al. Investigating macromolecules inside cultured and injected cells by in-cell NMR spectroscopy. Nat. Protoc. 1, 2701-2709 (2006).

6. Igarashi, R. et al. Distance determination in proteins inside Xenopus laevis oocytes by double electron-electron resonance experiments. J. Am. Chem. Soc. 132, 8228-8229 (2010).

7. Azarkh, M. et al. Long-range distance determination in a DNA model system inside Xenopus laevis oocytes by in-cell spin-label EPR. ChemBioChem 12, 1992-1995 (2011).

8. Krstic, I. et al. Long-range distance measurements on nucleic acids in cells by pulsed EPR spectroscopy. Angew. Chem. Int. Ed. 50, 5070-5074 (2011).

9. Singh, V., Azarkh, M., Exner, T.E., Hartig, J.S. \& Drescher, M. Human telomeric quadruplex conformations studied by pulsed EPR. Angew. Chem. Int. Ed. 48, 9728-9730 (2009).

10. Hänsel, R. et al. The parallel G-quadruplex structure of vertebrate telomeric repeat sequences is not the preferred folding topology under physiological conditions. Nucleic Acids Res. 39, 5768-5775 (2011)

11. Hänsel, R. et al. Evaluation of parameters critical for observing nucleic acids inside living Xenopus laevis oocytes by in-cell NMR spectroscopy. J. Am. Chem. Soc. 131, 15761-15768 (2009).

12. Azarkh, M. et al. Intracellular conformations of human telomeric quadruplexes studied by EPR. ChemPhysChem 13, 1444-1447 (2012).

13. Singh, V., Azarkh, M., Drescher, M. \& Hartig, J.S. Conformations of individual quadruplex units studied in the context of extended human telomeric DNA. Chem. Commun. 48, 8258-8260 (2012).

14. Edwards, T.E. \& Sigurdsson, S.Th. Site-specific incorporation of nitroxide spinlabels into 2'-positions of nucleic acids. Nat. Protoc. 2, 1954-1962 (2007).

15. Qin, P.Z. et al. Measuring nanometer distances in nucleic acids using a sequence-independent nitroxide probe. Nat. Protoc. 2, 2354-2365 (2007).

16. Ward, R., Keeble, D.J., El-Mkami, H. \& Norman, D.G. Distance determination in heterogeneous DNA model systems by pulsed EPR. ChemBioChem 8, 1957-1964 (2007)

17. Schiemann, 0., Cekan, P., Margraf, D., Prisner, T.F. \& Sigurdsson, S.Th. Relative orientation of rigid nitroxides of PELDOR: beyond distance measurements in nucleic acids. Angew. Chem. Int. Ed. 48, 3292-3295 (2009).
18. Marko, A. et al. Conformational flexibility of DNA. J. Am. Chem. Soc. 133, 13375-13379 (2011).

19. Sicoli, G. et al. Double electron-electron resonance (DEER): a convenient method to probe DNA conformational changes. Angew. Chem. Int. Ed. 47 735-737 (2008).

20. Sicoli, G. et al. Lesion-induced DNA weak structural changes detected by pulsed EPR spectroscopy combined with site-directed spin labeling. Nucleic Acids Res. 37, 3165-3176 (2009).

21. Kim, N.K., Bowman, M.K. \& DeRose, V.J. Precise mapping of RNA tertiary structure via nanometer distance measurements with double electron-electron resonance spectroscopy. J. Am. Chem. Soc. 132, 8882-8884 (2010).

22. Wunnicke, D. et al. Ligand-induced conformational capture of a synthetic tetracycline riboswitch revealed by pulse EPR. RNA 17, 182-188 (2011).

23. Reginsson, G.W. \& Schiemann, 0. Pulsed electron-electron double resonance: beyond nanometer distance measurements on biomacromolecules. Biochem. J. 434, 353-363 (2011).

24. Azarkh, M., Okle, 0., Eyring, P., Dietrich, D.R. \& Drescher, M. Evaluation of spin labels for in-cell EPR by analysis of nitroxide reduction in cell extract of Xenopus laevis oocytes. J. Magn. Reson. 212, 450-454 (2011).

25. Cekan, P., Smith, A.L., Barhate, N., Robinson, B.H. \& Sigurdsson, S.T. Rigid spin-labeled nucleoside C: a nonperturbing EPR probe of nucleic acid conformation. Nucleic Acids Res. 36, 5946-5954 (2008).

26. Barhate, N., Cekan, P., Massey, A.P. \& Sigurdsson, S.T. A nucleoside that contains a rigid nitroxide spin label: a fluorophore in disguise. Angew. Chem. Int. Ed. 46, 2655-2658 (2007).

27. Hustedt, E.J., Kirchner, J.J., Spaltenstein, A., Hopkins, P.B. \& Robinson, B.H. Monitoring DNA dynamics using spin-labels with different independent mobilities. Biochemistry 34, 4369-4375 (1995).

28. Shiokawa, K., Tashiro, K., Yamana, K. \& Sameshima, M. Electronmicroscopic studies of giant nucleus-like structure formed by lambda DNA introduced into the cytoplasm of Xenopus laevis fertilized-eggs and embryos. Cell. Differ. 20, 253-261 (1987).

29. Forbes, D.J., Kirschner, M.W. \& Newport, J.W. Spontaneous formation of nucleus-like structures around bacteriophage DNA microinjected into Xenopus eggs. Cell 34, 13-23 (1983).

30. Schnizler, K., Kuster, M., Methfessel, C. \& Fejtl, M. The Roboocyte: automated cDNA/mRNA injection and subsequent TEVC recording on Xenopus oocytes in 96-well microtiter plates. Receptors Channels 9, 41-48 (2003).

31. Jeschke, G. DeerAnalysis2011 user manual (2011) http://www.epr.ethz.ch/ software/DeerAnalysis2011_manual.pdf.

32. Chiang, Y.M., Borbat, P.P. \& Freed, J.H. The determination of pair distance distributions by pulsed ESR using Tikhonov regularization. J. Magn. Reson. 172, 279-295 (2005)

33. Jeschke, G. et al. DeerAnalysis2006-a comprehensive software package for analyzing pulsed ELDOR data. Appl. Magn. Reson. 30, 473-498 (2006).

34. Jeschke, G. \& Polyhach, Y. Distance measurements on spin-labelled biomacromolecules by pulsed electron paramagnetic resonance. Phys. Chem. Chem. Phys. 9, 1895-1910 (2007).

35. Dumont, J.N. Oogenesis in Xenopus laevis (Daudin). I. Stages of oocyte development in laboratory maintained animals. J. Morphol. 136, 153-179 (1972).

36. Jeschke, G., Pannier, M. \& Spiess, H.W. Distance measurements in biological systems by EPR. in Distance Measurements in Biological Systems by EPR, Vol. 19 (eds. Berliner, L.J., Eaton, G.R. \& Eaton, S.S.) 493-511 (Kluwer Academic/Plenum Publishers, 2000).

37. Spaltenstein, A., Robinson, B.H. \& Hopkins, P.B. Sequence-dependent and structure-dependent DNA-base dynamics - Synthesis. Structure, and dynamics of site and sequence specifically spin-labeled DNA. Biochemistry 28, 9484-9495 (1989).

38. Stork, S.W. \& Makinen, M.W. Facile synthesis of 3-formyl-2,2,5,5tetramethyl-1-oxopyrroline. Synthesis 1999, 1309-1312 (1999). 